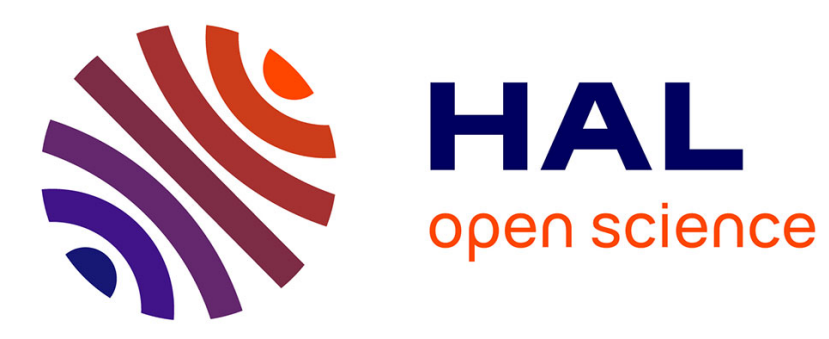

\title{
Inhomogeneity and Lagrangian unsteadiness in turbulent thermal convection
}

Olivier Liot, Amélie Gay, Julien Salort, Mickaël Bourgoin, Francesca Chillà

\section{To cite this version:}

Olivier Liot, Amélie Gay, Julien Salort, Mickaël Bourgoin, Francesca Chillà. Inhomogeneity and Lagrangian unsteadiness in turbulent thermal convection. Physical Review Fluids, 2016, 1, pp.064406. 10.1103/PhysRevFluids.1.064406 . hal-01506986

\section{HAL Id: hal-01506986 https://hal.science/hal-01506986}

Submitted on 12 Apr 2017

HAL is a multi-disciplinary open access archive for the deposit and dissemination of scientific research documents, whether they are published or not. The documents may come from teaching and research institutions in France or abroad, or from public or private research centers.
L'archive ouverte pluridisciplinaire HAL, est destinée au dépôt et à la diffusion de documents scientifiques de niveau recherche, publiés ou non, émanant des établissements d'enseignement et de recherche français ou étrangers, des laboratoires publics ou privés. 


\title{
On inhomogeneity and Lagrangian unsteadiness in turbulent thermal convection
}

\author{
Olivier Liot, ${ }^{*}$ Amélie Gay, Julien Salort, Mickaël Bourgoin, and Francesca Chillà ${ }^{\dagger}$ \\ Univ Lyon, ENS de Lyon, Univ Claude Bernard, CNRS, \\ Laboratoire de Physique, F-69342 Lyon Cedex 7, France
}

(Dated: November 10, 2016)

\begin{abstract}
We present an experimental study of non-homogeneous turbulence using a Rayleigh-Bénard convection cell. The fluid motion is forced by a temperature difference between two horizontal plates. Using Lagrangian tracking on a large volume we can capture part of the Large Scale Circulation. The velocity statistics are strongly affected by the inhomogeneous mean flow but we recover the typical Homogeneous Isotropic Turbulence statistics by removing the local average. We discuss and explain a Lagrangian unsteadiness which persists because of the Large Scale Circulation oscillations. Our Lagrangian approach is a new way to study specificities of the convective roll motions in turbulent thermal convection. We propose a model based on the convolution between the Large Scale Circulation oscillations and the turbulent fluctuations to explain the shape of the velocity PDFs. However, the acceleration statistics are not affected by the mean flow.
\end{abstract}

\section{INTRODUCTION}

Understanding the properties of turbulent transport which occurs in thermal flows is still a challenge. Many natural (atmospheric and oceanic dynamics, processes in planetary cores) and industrial (cooling of buildings, heat exchangers) flows are indeed controlled by thermal convection without mechanical forcing. The most common used laboratory model system is the Rayleigh-Bénard cell. A layer of fluid confined in a closed cell is cooled from above and heated from below. If the thermal forcing is sufficient, a turbulent flow can appear. Temperature gradients are confined into the thermal boundary layers close to the top and bottom plates. Their destabilization implies the development of fluid pockets called thermal plumes. During the last two decades, much progress were made to understand the coupling between thermal forcing and heat flux across the cell or the turbulent properties of velocity and temperature fields [1-3]. Nevertheless, numerous points are still open to discussion like plume dynamics, boundary layers structure [4] or interactions between large scale flow and fluctuations.

Improvements of techniques and computing resources during the last decade allow high-resolution spatiotemporal Lagrangian measurements in turbulent flows $[5,6]$. This point of view is very adapted to flows with important mixing [7]. Moreover, some facets of the turbulence as finite-Reynolds effects are described with stochastic models using Lagrangian approach $[8,9]$. The Lagrangian tracking of particles opens the door of multiparticle statistics [10]. Although experimental and numerical Lagrangian studies are well developed for classic turbulence [11], it is not the case for thermal convection. To our knowledge, there is one large numerical study of Lagrangian transport in thermal convection $[12,13]$. Two different Lagrangian experiments were

\footnotetext{
* Presently at Departamento de física y matemática aplicada, Universidad de Navarra, c/ Irunlarrea, Pamplona, Spain

$\dagger$ francesca.chilla@ens-lyon.fr
}

also proposed. The first one, in Lyon, was made using a large particle with embedded temperature sensors which was immersed in a Rayleigh-Bénard cell [14]. Recent improvements of the technique have led to robust statistics [15]. This instrumented particle is able to explore all the flow but finite-size effects are observed. The second one, in Hong-Kong, explored the very center of convection cells - where the flow is quite homogeneous - with sub-millimetric particles [16, 17]. To be complementary, we propose to combine sub-millimetric particle tracking and observation of a large part of the flow.

In this letter we present a new experiment of Lagrangian tracking in a turbulent Rayleigh-Bénard cell. We propose to introduce a first bridge between the HongKong studies and the Lyon ones. The measurement volume is wide enough to observe part of the Large Scale Circulation (LSC). We are deliberately out of the Homogeneous Isotropic Turbulence (HIT) framework. Indeed we want to observe the impact of the LSC on turbulent statistics of tracers. This letter focuses on one-particle statistics (in opposition to two-particle statistics as pair dispersion). We will discuss both the effects of the inhomogeneity and unsteadiness of the LSC structure on the velocity statistics. Then we will present the acceleration ones.

\section{EXPERIMENTAL SETUP AND MEASUREMENT TECHNIQUES}

Our experimental setup is an octagonal cell (figure 1) filled with deionized water with a height of $H=30 \mathrm{~cm}$, an inner diameter of $D=40 \mathrm{~cm}$. The top and bottom plates are made of anodized aluminium. We impose the heat flux from the bottom with a heating electrical resistance. The top plate temperature is fixed with a regulated glycol circulation. The walls are made of polymethylmetacrylate (PMMA). The imposed heat flux is $600 \mathrm{~W}$ and the top plate is maintained at $30.4^{\circ} \mathrm{C}$. The resulting difference of temperature is $\Delta T=19.2^{\circ} \mathrm{C}$. The Rayleigh number is therefore $R a=g \alpha \Delta T H^{3} / \nu \kappa=2.0 \times 10^{10}$, 


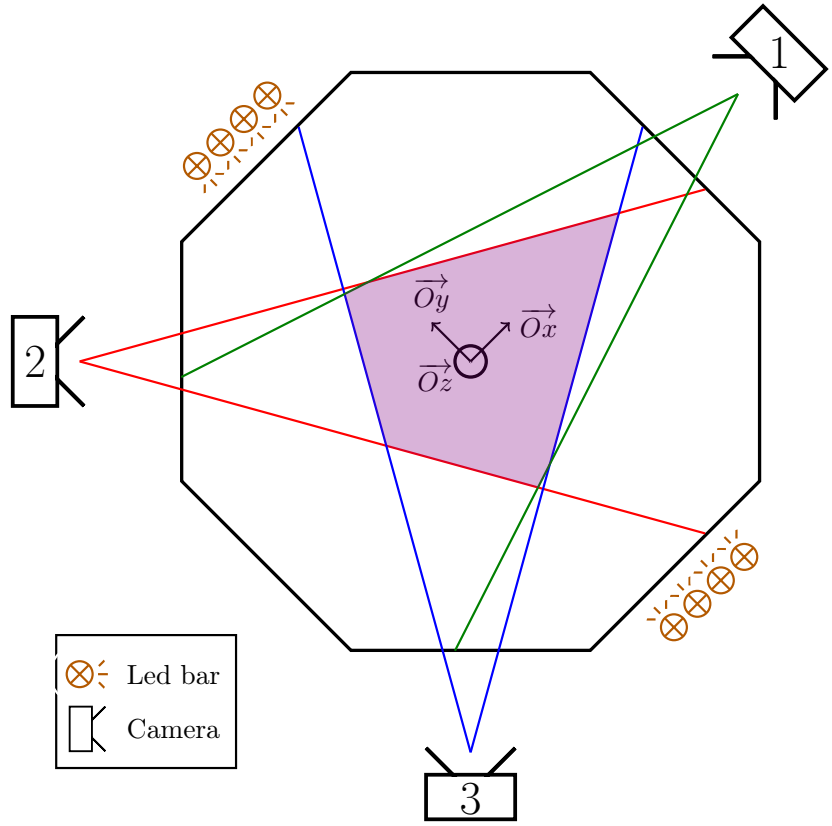

FIG. 1. Top-view of the convection cell and measurement setup. The purple area corresponds to a horizontal slice of the measurement volume observed by the three cameras. See text for more details about the dimensions and camera specifications.

where $g$ is the acceleration due to gravity, $\alpha$ the thermal expansion coefficient of water, $\nu$ its kinematic viscosity and $\kappa$ its thermal diffusivity. The Nusselt number is $N u=Q H / \lambda \Delta T \approx 100$ (including about $15 \%$ of losses) where $Q$ represents the heat flux across the cell and $\lambda$ the thermal conductivity of water. The Prandtl number, defined as $\operatorname{Pr}=\nu / \kappa$, reaches 4.4 for a mean temperature of $40^{\circ} \mathrm{C}$. In these conditions the convective flow is turbulent. A roll confined between two diametrically opposite sides of the lateral walls appears with a turnover time of about $80 \mathrm{~s}$. We have qualitatively observed using shadowgraphy that it can change spontaneously of sidewalls pair between which it is fixed with a typical time scale of some hours. This LSC is indeed known to exhibit a long time dynamics with spontaneous changes of position and orientation like reversals or cessations (see e.g. $[2,18,19])$. The large time scale of the flow is much longer than the observation time of the experiments reported here (six runs of $180 \mathrm{~s}$ ) and its study goes beyond the scope of the present work.

Three coplanar pr $1088 \times 2048$ pixels $^{2}$ cameras are placed around the cell at equal vertical distance from both plates and at polar angles $\psi=45^{\circ}, 180^{\circ}$ and $270^{\circ}$ (respectively numbered 1 to 3 on the figure 1). We perform a 3D Lagrangian tracking in a measurement volume of approximately $11 \mathrm{~cm}$ aside and $17 \mathrm{~cm}$ in height centered in the cell. It corresponds to a ratio of about 6 pixels per Kolmogorov length $-\eta=\left(\nu^{3} / \epsilon\right)^{1 / 4} \approx 500 \mu \mathrm{m}$ where $\epsilon$ is the mass rate of kinetic energy dissipation. We estimate the energy dissipation as $\epsilon=\operatorname{RaPr}^{-2}(\mathrm{Nu}-$ 1) $\nu^{3} / H^{4}[20]$. We seed the flow with polystyrene particles with a diameter of $250 \mu \mathrm{m}$ and density of $1.03 \mathrm{~g} / \mathrm{cm}^{3}$. These particles are illuminated using 8 vertical bars of 6 white LEDs with a brightness of 864 lumens (see the figure 1 for the spatial disposition). We perform sets of six 180 -second acquisitions. The total measurement time of one set corresponds to about 2700 times the Kolmogorov time scale $-\tau_{\eta}=\sqrt{\nu / \epsilon} \approx 0.4 \mathrm{~s}-$ and 540 times the free-fall one $-\tau_{f f}=\sqrt{H / g \alpha \Delta T}$ - so the flow is well averaged for time scales to which the LSC remains fixed between two opposite sidewalls. The sampling frequency must be large enough compared to the Kolmogorov time scale to resolve dissipative scales. Besides, oversampling is compulsory to resolve and properly filter measurements noise (for instance due to the refractive index thermal fluctuations [16]) from the reconstructed trajectories [21] and estimate the velocity and acceleration statistics. As empirically suggested by previous Lagrangian tracking experiments to accurately measure velocity and acceleration [6],we use a sampling frequency of $200 \mathrm{~Hz}$, hence oversampling by a factor of 80 the dissipative time-scale. The trajectories and their temporal derivatives are filtered using the traditional method of convolution with a Gaussian kernel of width $0.3 \tau_{\eta}$ which does not affect the dissipative scale resolution.

\section{VELOCITY STATISTICS}

First we present in the figure 2 the probability density functions (PDFs) of the three components of the Lagrangian velocity. For the HIT case we expect Gaussian PDFs. Nevertheless the two horizontal components are found to be highly non-Gaussian. As we will see this is related to the fact that the Lagrangian statistics are affected by the inhomogeneity of the flow. In our measurement volume, the large scale flow is principally horizontal and highly inhomogeneous. The figure 3 illustrates this point by showing the $y$ component mean Eulerian field $v_{y}^{E}$ of the flow obtained by binning the space and averaging the Lagrangian data in each bin. We observe the signature of the convection roll present in the cell. The axes are chosen $a d$ hoc to match the $x$ axis with the rotation axis of the roll. Consequently the $x$ component mean velocity $v_{x}^{E}$ is nearly null (not shown here). The measurement volume is higher than wide so that we do not really see the vertical mean flow confined close to the vertical walls (see inset figure 3 ).

To put forward the influence of the mean flow on the Lagrangian statistics, we split up the velocity into a mean local velocity at the particle position $\left(v_{k}^{E}\right)$ and a fluctuating Lagrangian one $\left(v_{k}^{\prime}\right)$ :

$$
v_{k}(t)=v_{k}^{E}(x(t), y(t), z(t))+v_{k}^{\prime}(t),
$$

where $k=x, y, z$. The figure 4 shows the PDF of each 


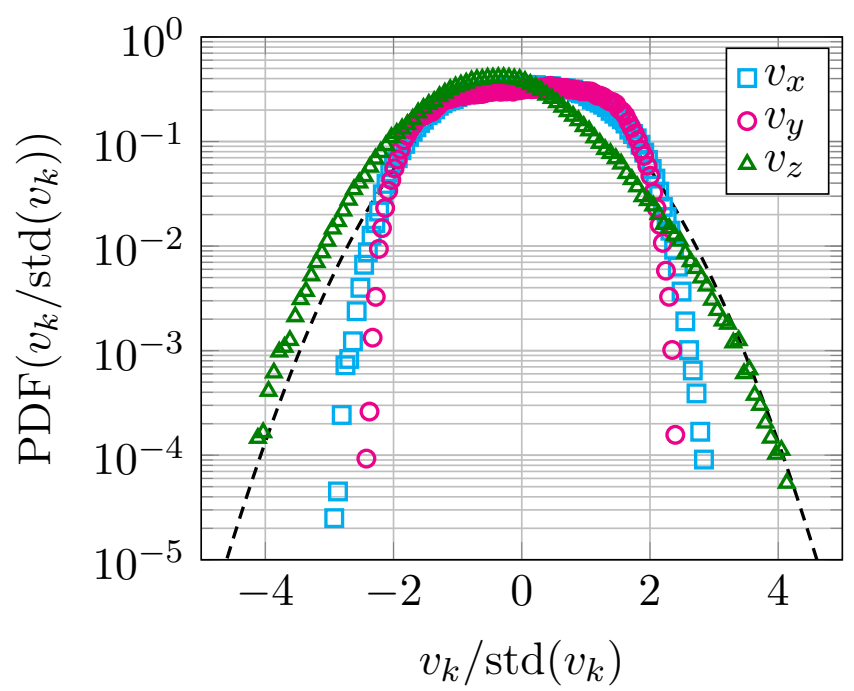

FIG. 2. PDFs of the velocity components $v_{k}$ normalized by the corresponding standard deviation. The dashed line represents a Gaussian distribution.

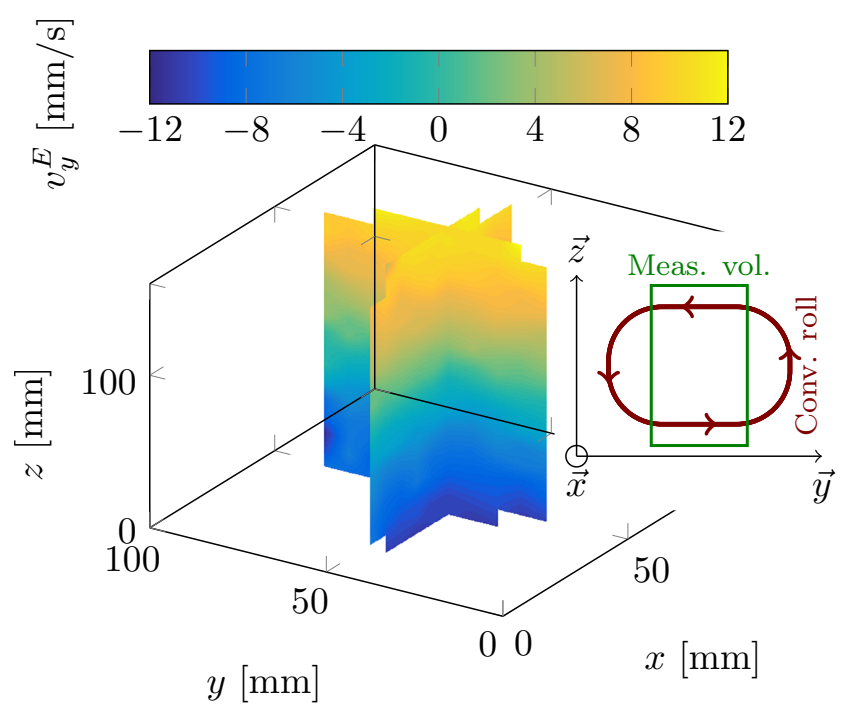

FIG. 3. Vertical slices of the mean Eulerian velocity $v_{y}^{E}$. Inset: sketch of the convection roll and of the measurement volume in the $\{\vec{y}, \vec{z}\}$ plan.

component of the fluctuating velocity $v_{k}^{\prime}$. We observe that the $v_{z}^{\prime}$ and $v_{y}^{\prime}$ PDFs collapse on a Gaussian distribution. The non-Gaussianity seen in the figure 2 for $v_{y}$ and $v_{z}$ was therefore entirely related to the large inhomogeneity of the mean flow due to the LSC. However, the $v_{x}^{\prime} \mathrm{PDF}$ is nearly unchanged compared to the $v_{x}$ one. As previously pointed, the $x$-axis was chosen to be aligned with the rotation axis of the convection roll, so that the mean field $v_{x}^{E}$ is nearly null. This brings the following paradox: (i) since $v_{x}^{E}$ is null, according to eq. 1 it is

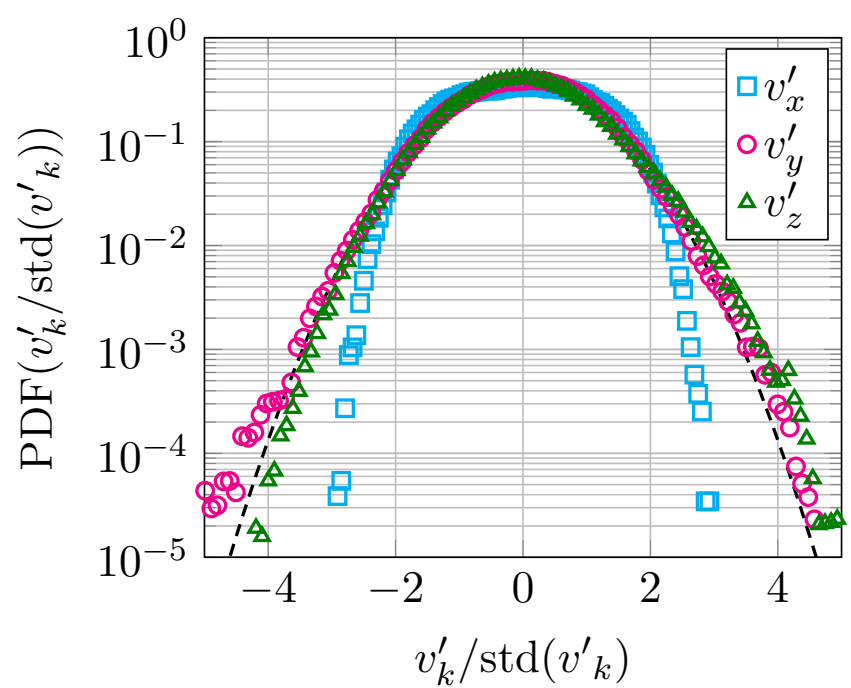

FIG. 4. PDFs of the three fluctuating velocity components $v_{k}^{\prime}(t)$ normalized by the corresponding standard deviation. The dashed line represents a Gaussian distribution.

expected that $v_{x}(t) \approx v_{x}^{\prime}(t)$ so if the PDF of $v_{x}$ is nonGaussian, so should be $v_{x}^{\prime}$; (ii) however, the analysis on $v_{y}$ and $v_{z}$ suggests that the non-Gaussianity arises because of the LSC, so in the absence of a $x$ component mean velocity, one could expect $x$ component fluctuations to be Gaussian. This paradox can be solved by considering there are two sources of Lagrangian unsteadiness. First in the Lagrangian framework the large scale inhomogeneity of $v^{E}(x, y, z)$ results in a non-stationary Lagrangian perturbation $v^{L}(t)=v^{E}(x(t), y(t), z(t))$ along the particle trajectory $(x(t), y(t), z(t))$ as seen in eq. 1. This unsteadiness has been taken care of by removing from the Lagrangian trajectories the local Eulerian average. Then the remaining non-Gaussianity of $v_{x}^{\prime}$ results from an intrinsic unsteadiness of the flow very likely to be attributed to small motions of the convection roll. The roll is confined between two opposite sides of the cell (at the time scale of our acquisitions). But it could still weakly oscillate at sufficient short time scales to impact the velocity statistics without being visible on the average. To confirm this explanation, we show in the figure 5 the ratio $v_{x}^{\prime} R M S / v_{y}^{\prime} R M S$ where $R M S$ means the root-mean square. In statistically stationary conditions, we should expect a ratio close to one, as for both horizontal components the effect of thermal plumes on velocity fluctuations should be similar. On the contrary, $v_{x}^{\prime} R M S$ is about $50 \%$ higher than $v_{y}^{\prime} R M S$ which can be attributed to the signature of roll motions.

Mathai et al. [22] have observed very similar velocity PDFs for suspended particles in a turbulent system with a non-null mean flow. They attribute this to a periodicity in the velocity induced by the particle interaction with the mean flow. In our case it is a little bit different because such a velocity periodicity is likely due to the 


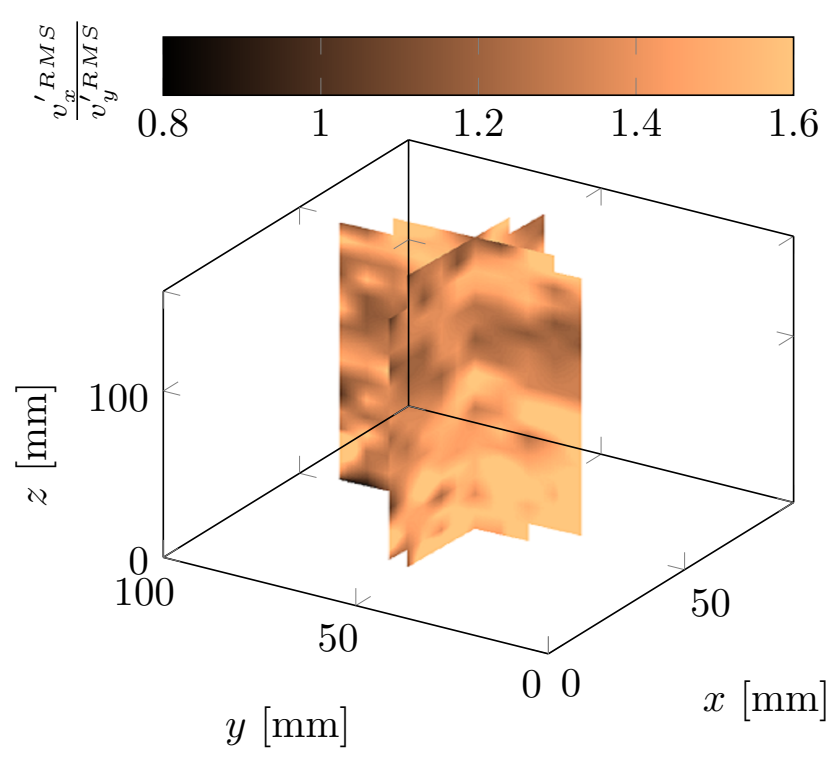

FIG. 5. Vertical slices of the ratio $v_{x}^{\prime R M S} / v_{y}^{\prime R M S}$.

mean flow oscillation itself. But the resulting velocity statistics should be similar. Indeed we probably observe the so-called sloshing motion of the convection roll [23$25]$ in our cell. We propose a simple model to explain the shape of the PDF of $v_{x}^{\prime}$. The ratio $v_{x}^{\prime R M S} / v_{y}^{\prime} R M S$ is quite homogeneous so we consider at the first order that the convection roll motion is an oscillating solid translation along the $x$-axis. A diagram is presented in the inset of the figure 6 . We call $\overrightarrow{V_{\text {inst }}}$ the velocity induced by the roll motions. At every time the roll center position is called $X(t)$ and we have $\overrightarrow{V_{\text {inst }}}(t)=\dot{X}(t) \vec{x}$. With this model the $v_{y}$ velocity is not affected by the roll oscillation. Velocities $V_{\text {inst }}(t)$ are in a range $\left[-V_{\max }, V_{\max }\right]$. At the first order we assume that all velocities have the same probability to occur in this range. For the example of a periodic oscillation with amplitude $X_{0}$ and pulsation $\omega$ we have $V_{\max }=X_{0} \omega$. If we assume that in absence of roll oscillation the PDF of $v_{x}^{\prime}$ would be a centered Gaussian with standard deviation $\sigma$ (related to turbulent fluctuations), the PDF induced by the roll oscillation would be simply obtained by the convolution of this Gaussian with a centered rectangular function of width $2 V_{\max }$. The resulting fit equation is the convolution product $(*)$ :

$$
\begin{aligned}
\operatorname{PDF}^{f i t}\left(v_{x}^{\prime}\right)= & {\left[\frac{1}{\sqrt{2 \pi} \sigma} \exp \left(\frac{-v_{x}^{\prime 2}}{2 \sigma^{2}}\right)\right] * } \\
& {\left[\operatorname{erf}\left(\frac{v_{x}^{\prime}+V_{\text {max }}}{V_{\text {norm }}}\right)-\operatorname{erf}\left(\frac{v_{x}^{\prime}-V_{\text {max }}}{V_{\text {norm }}}\right)\right], }
\end{aligned}
$$

We can fit the experimental PDF of $v_{x}^{\prime}$ shown in figure 4 with this expression. For the ease of fit we use sharp error functions to build our rectangular distribution. The error function sharpness are fixed with
$V_{\text {norm }}=0.01 \mathrm{~mm} / \mathrm{s}$ whose does not affect the shape of the rectangular distribution for $V_{\text {norm }} \ll 1 \mathrm{~mm} / \mathrm{s}$. The figure 6 compares the best fit from eq. 2 with the PDF of $v_{x}^{\prime}$ with fit parameters in good agreement with the phenomenon scales. These ones reach $\sigma=2.8 \pm 0.1 \mathrm{~mm} / \mathrm{s}$ and $V_{\max }=8.7 \pm 0.1 \mathrm{~mm} / \mathrm{s} . \sigma$ is a bit smaller than the standard deviation of $v_{y}^{\prime}$ and $v_{z}^{\prime}$ which reach respectively $4.1 \mathrm{~mm} / \mathrm{s}$ and $3.8 \mathrm{~mm} / \mathrm{s}$. This difference perhaps denotes an anisotropy due to the high velocities in the $\vec{y}$ direction. With the value of $V_{\max }$ we estimate the typical time scale of the roll motions. Considering the octagonal shape of the cell, the maximal amplitude for the roll translation is estimated to be in a range between a quarter and a half of one sidewall width - i.e. between 3.5 and $7 \mathrm{~cm}$. Therefore, if we assume a periodic oscillation, and the relation $V_{\max }=X_{0} \omega$, the fitted value for $V_{\max }$ corresponds to a pulsation $\omega \in[0.12,0.25] \mathrm{rad} / \mathrm{s}$, i.e. an oscillation period in the range $[26-51] \mathrm{s}$ which is significantly less than the acquisition time (six runs of $180 \mathrm{~s}$ ). This is consistent with the fact that the roll oscillation is averaged while computing $v_{x}^{E}$. Furthermore it is in agreement with previous LSC oscillation time scale measured at $R a=6.5 \times 10^{9}$ and $\operatorname{Pr}=5.3[26]$.

This sloshing has not been observed by Ni et al. [16]. Their velocity PDFs obtained in the very center of the convection cell are very Gaussian whereas the sloshing should also occurs in their cell. This difference could be due to geometric effects. In our cell the convection roll is confined between two opposite flat walls whereas the $\mathrm{Ni}$ et al. cell is perfectly cylindrical. Because they put up less resistance to a longitudinal motion compared to cylindrical walls, the flat sidewalls could allow sufficiently important sloshing to be detected on our velocity statistics contrary to the $\mathrm{Ni}$ et al. ones [16].

\section{ACCELERATION STATISTICS}

We study now the acceleration statistics. The figure 7 shows the normalized PDFs of the three acceleration components and the one of the vertical acceleration obtained by $\mathrm{Ni}$ et al. in the very center of a convection cell (i.e. with very few effects of inhomogeneity) [16]. All distributions have a similar stretched exponential shape, which is common for the Lagrangian acceleration in classic turbulent flows $[6,11,21]$. The acceleration standard deviations reach respectively $2.5 \mathrm{~mm} / \mathrm{s}^{2}, 2.3 \mathrm{~mm} / \mathrm{s}^{2}$ and $2.0 \mathrm{~mm} / \mathrm{s}^{2}$ for $a_{x}, a_{y}$ and $a_{z}$ resepctively. The PDF shape is in a good agreement with previous experimental [16] and numerical [12] works in turbulent thermal convection. To confirm this point we compare the acceleration variance to the kinetic energy dissipation rate. If we observe a space-time region small enough, the acceleration variance can be linked to the kinetic energy dissipation rate $[6,27]$ :

$$
\left(\operatorname{std}\left(a_{k}\right)\right)^{2}=a_{0} \epsilon^{3 / 2} \nu^{-1 / 2}
$$




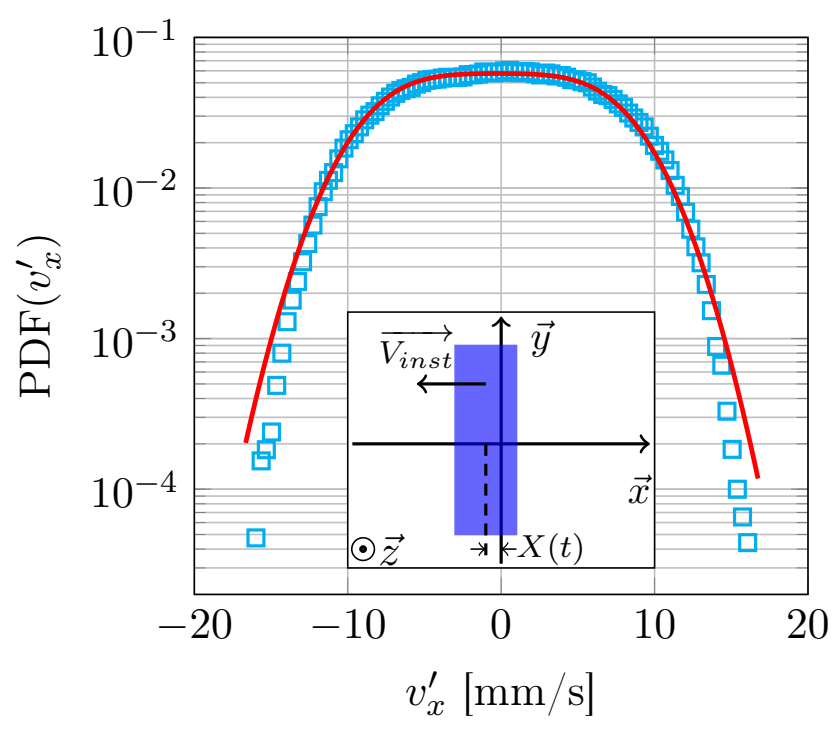

FIG. 6. PDF of $v_{x}^{\prime}$. The solid line is the normalized fit from eq. 2. Inset: scheme of the velocity $\overrightarrow{V_{\text {inst }}}(t)$ induced by the roll translation. The purple region represents the top view of the roll around the $z$-axis.

where $k=x, y, z$ and $a_{0}$ is supposed to be an universal constant. The $\epsilon$ value derived from the global quantities (see the setup description and [20]) does not take into account the inhomogeneity of the flow. The numerical study of Kunnen et al. [28] shows that the value of $\epsilon$ higly depends on the localisation in the flow. Using their maps of $\epsilon$ in a cylindrical cell we estimate the kinetic energy dissipation rate in our measurement volume between $30 \%$ and $40 \%$ of the one computed from global quantities. For the vertical acceleration we obtain $a_{0}=2.8 \pm 0.5$. We compare this value to the systematic study performed by $\mathrm{Ni}$ et al. [16] for different Rayleigh numbers. The expected value for $\operatorname{Pr}=4.4$ ranges between around 2.5 so our measurement is in quite good agreement with this study.

Nevertheless, the numerical simulations from Schumacher [12] $\left(R a=1.2 \times 10^{8}, \operatorname{Pr}=0.7\right)$ show an anisotropy of the acceleration with wider PDFs for the horizontal accelerations. This can be related to the plumes vorticity [29]. In our experiments we do not capture the lateral jets where the plumes are concentrated what can explain why we don't observe this anisotropy. Contrary to the velocity, the acceleration is not affected by the mean flow. It does exhibit a slightly inhomogeneous mean acceleration field (especially for $a_{z}$ ) but the fluctuations reach several tens of the mean acceleration field (not shown here) and the PDFs are not affected. Moreover this robustness of acceleration statistics is very likely to be related to the small correlation time $(\sim 1 \mathrm{~s})$ and correlation length of acceleration components compared to the typical scales of the flow inhomogeneity and unsteadiness.

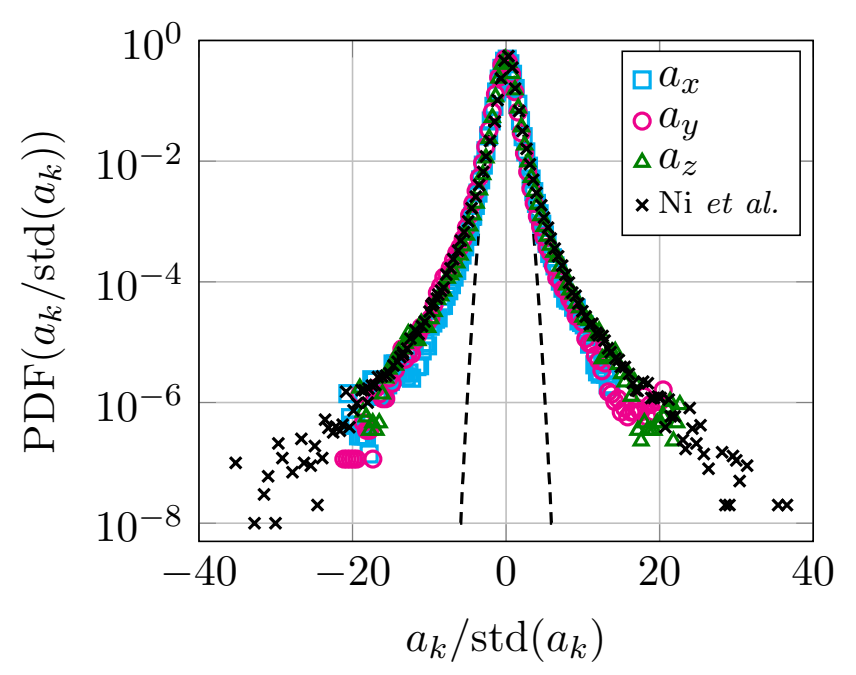

FIG. 7. Normalized PDFs of $a_{k}$. The black crosses represent the PDF of $a_{z}$ obtained by Ni et al. [16] $\left(R a=4.3 \times 10^{9}\right.$, $\operatorname{Pr}=6.1)$.

\section{DISCUSSION AND CONCLUSION}

We have proposed a Lagrangian experiment to explore turbulent statistics in a large part of a convection cell with two aims: to study the inhomogeneity of the LSC and the roll motions. By observing a wider area than the very center of a turbulent convection cell, we have shown that the Lagrangian statistics are differently affected by the mean flow imposed by the wall confinement. The velocity distributions are very far from the well-known HIT Gaussian shape. By removing the contribution of the mean flow we recover Gaussian PDFs, except for one velocity component affected by the motions of the convection roll. The low number of plumes in the center of the cell - compared to the falling and rising jets - explain that we observe very these Gaussian PDFs. We propose a very simple model based on an oscillating translation of the LSC to explain the shape of the PDF of $v_{x}^{\prime}$ which takes into account the convolution between the turbulent fluctuations and the additional perturbations induced by the convection roll oscillation. The acceleration statistics are similar to the previous Lagrangian studies and are not affected by the mean flow. This study showed two aspects of the Lagrangian unsteadiness. An inhomogeneous Eulerian mean field imposes an unsteadiness along the trajectory of the particle which can be removed by simply subtracting the local Eulerian mean field along the Lagrangian path of tracers. Alternatively this shows how the remaining non-Gaussianity (after local inhomogeneity has been removed) can be advantageously used to infer subtler properties of the flow phenomenology. Here for instance, we could infer the typical time scale of roll oscillations from the velocity PDFs coupled to a simple model. Some improvements in the tracking technique 
would be very interesting to observe a wider volume and track particles over a longer time in order for instance to explicitly investigate the roll dynamics or the Lagrangian correlations. The area close to the walls where the thermal plumes are concentrated is an other zone of investigation where some specificities of the turbulent convection could be observed. We plan also to study the particle transport, using pair dispersion, to investigate peculiari- ties of an inhomogeneous turbulent flow.

\section{ACKNOWLEDGMENTS}

We gratefully thank Marc Moulin for the manufacture of the cell and Miguel Lopez-Caballero for his help with the camera calibration.
[1] S. Grossmann and D. Lohse, "Scaling in thermal convection: a unifying theory," Journal of Fluid Mechanics 407, 27-56 (2000).

[2] G. Ahlers, S. Grossmann, and D. Lohse, "Heat transfer and large scale dynamics in turbulent Rayleigh-Bénard convection," Reviews of modern physics 81, 503 (2009).

[3] D. Lohse and K.-Q. Xia, "Small-scale properties of turbulent Rayleigh-Bénard convection," Annual Review of Fluid Mechanics 42, 335-364 (2010).

[4] F. Chillà and J. Schumacher, "New perspectives in turbulent Rayleigh-Bénard convection," The European Physical Journal E: Soft Matter and Biological Physics 35, $1-25$ (2012).

[5] A. La Porta, G. A. Voth, A. M. Crawford, J. Alexander, and E. Bodenschatz, "Fluid particle accelerations in fully developed turbulence," Nature 409, 1017-1019 (2001).

[6] G. A. Voth, A. La Porta, A. M. Crawford, J. Alexender, and E. Bodenschatz, "Measurement of particle accelerations in fully developped turbulence," Journal of Fluid Mechanics 469, 121-160 (2002).

[7] R. O. Fox and P. K. Yeung, "Improved lagrangian mixing models for passive scalars in isotropic turbulence," Physics of Fluids 15, 961-985 (2003).

[8] B. L. Sawford, "Reynolds number effects in lagrangian stochastic models of turbulent dispersion," Physics of Fluids A: Fluid Dynamics 3, 1577-1586 (1991).

[9] Stephen B Pope, "Stochastic lagrangian models of velocity in homogeneous turbulent shear flow," Physics of Fluids 14, 1696-1702 (2002).

[10] M. Bourgoin, N. T. Ouellette, H. Xu, J. Berg, and E. Bodenschatz, "The role of pair dispersion in turbulent flow," Science 311, 835-838 (2006).

[11] F. Toschi and E. Bodenschatz, "Lagrangian properties of particles in turbulence," Annual Review of Fluid Mechanics 41, 375-404 (2008).

[12] Jörg Schumacher, "Lagrangian dispersion and heat transport in convective turbulence," Phys. Rev. Lett. 100, 134502 (2008).

[13] Jörg Schumacher, "Lagrangian studies in convective turbulence," Phys. Rev. E 79, 056301 (2009).

[14] Y. Gasteuil, W. L. Shew, M. Gibert, F. Chilla, B. Castaing, and J.-F. Pinton, "Lagrangian temperature, velocity, and local heat flux measurement in Rayleigh-Bénard convection," Physical Review Letters 99, 234302 (2007).

[15] O. Liot, F. Seychelles, F. Zonta, S. Chibbaro, T. Coudarchet, Y. Gasteuil, J.-F. Pinton, J. Salort, and F. Chillà, "Simultaneous temperature and velocity Lagrangian measurements in turbulent thermal convection," Journal of Fluid Mechanics 794, 655-675 (2016).

[16] R. Ni, S.-D. Huang, and K.-Q. Xia, "Lagrangian acceleration measurements in convective thermal turbulence,"
Journal of Fluid Mechanics 692, 395-419 (2012).

[17] Rui Ni and Ke-Qing Xia, "Experimental investigation of pair dispersion with small initial separation in convective turbulent flows," Physical Review E 87, 063006 (2013).

[18] Francisco Fontenele Araujo, Siegfried Grossmann, and Detlef Lohse, "Wind reversals in turbulent rayleighbénard convection," Physical review letters 95, 084502 (2005).

[19] Heng-Dong Xi and Ke-Qing Xia, "Cessations and reversals of the large-scale circulation in turbulent thermal convection," Physical Review E 75, 066307 (2007).

[20] B. I. Shraiman and E. D. Siggia, "Heat transport in high-Rayleigh-number convection," Physical Review A 42, 3650 (1990).

[21] N. Mordant, A. M. Crawford, and E. Bodenschatz, "Experimental lagrangian acceleration probability density function measurement," Physica D: Nonlinear Phenomena 193, 245-251 (2004).

[22] Varghese Mathai, Vivek N Prakash, Jon Brons, Chao Sun, and Detlef Lohse, "Wake-driven dynamics of finitesized buoyant spheres in turbulence," Physical review letters 115, 124501 (2015).

[23] X-L Qiu, X-D Shang, Penger Tong, and K-Q Xia, "Velocity oscillations in turbulent rayleigh-bénard convection," Physics of Fluids 16, 412-423 (2004).

[24] Quan Zhou, Heng-Dong Xi, Sheng-Qi Zhou, Chao Sun, and Ke-Qing Xia, "Oscillations of the large-scale circulation in turbulent rayleigh-bénard convection: the sloshing mode and its relationship with the torsional mode," Journal of Fluid Mechanics 630, 367-390 (2009).

[25] Heng-Dong Xi, Sheng-Qi Zhou, Quan Zhou, Tak-Shing Chan, and Ke-Qing Xia, "Origin of the temperature oscillation in turbulent thermal convection," Physical review letters 102, 044503 (2009).

[26] Heng-Dong Xi, Quan Zhou, and Ke-Qing Xia, "Azimuthal motion of the mean wind in turbulent thermal convection," Physical Review E 73, 056312 (2006).

[27] A.S. Monin and A.M. Yaglom, Statistical fluid mechanics: mechanics of turbulence (Dover, 2007).

[28] R. P. J. Kunnen, H. J. H. Clercx, B. J. Geurts, L. J. A. van Bokhoven, R. A. D. Akkermans, and R. Verzicco, "Numerical and experimental investigation of structurefunction scaling in turbulent Rayleigh-Bénard convection," Physical Review E 77, 016302 (2008).

[29] Q. Zhou, C. Sun, and K.-Q. Xia, "Morphological evolution of thermal plumes in turbulent Rayleigh-Bénard convection," Physical Review Letters 98, 074501 (2007). 